\title{
Intertwined $\alpha \beta$ Spectrin Meeting Helical Actin Protofilament in the Erythrocyte Membrane Skeleton: Wrap-Around vs. Point-Attachment
}

\author{
Paul Sche, Carlos Vera, and L. Amy Sung \\ Department of Bioengineering, Jacobs School of Engineering, University of California, La Jolla, San Diego,
} CA 92093-0412, USA

(Received 23 November 2010; accepted 3 March 2011; published online 17 March 2011)

Associate Editor Cheng Dong oversaw the review of this article.

\begin{abstract}
Our 3-D model for a junctional complex (JC) in the erythrocyte membrane skeleton proposed that the helical actin protofilament functions as a mechanical axis for three pairs of $\alpha \beta$ spectrin (Sp), and each pair wraps around the protofilament in a back-to-back fashion. The distal end of each $\mathrm{Sp}$ is further associated with the lipid bilayer by a suspension complex (SC). Here, we detail how splitting and rejoining of $\alpha \beta \mathrm{Sp}$ around a protofilament may form a loop that sustains and equilibrates tension. Sequential association of $\beta$ and $\alpha$ Sp solves the challenge of constructing multiple loops along the protofilament, and topological connection facilitates their re-association. The wrap-around model minimizes the strain of the actin binding site on $\beta \mathrm{Sp}$ due to tension, redirection, or sliding of intertwined Sp. Pairing $\mathrm{Sp}$ balances the opposing forces and provides a mechanism for elastic recovery. The wrap-around junction thus provides mechanical advantages over a point-attachment junction in maintaining the integrity and functionality of the network. Severing $\alpha$ or $\beta \mathrm{Sp}$ may convert a wrapping-around junction to a point-attachment junction. In that case, a "bow up" motion of JC during deformation may disturb or flip the overlaid lipid bilayer, and mark stressed erythrocytes for phagocytosis.
\end{abstract}

Keywords-Red blood cell, Deformation, Junctional complex, Death signals.

\section{INTRODUCTION}

Circulating erythrocytes exchange $\mathrm{O}_{2}$ and $\mathrm{CO}_{2}$ as they pass through small capillaries in tissues. Their essential elastic deformability is governed by a hexagonal protein skeletal network underneath the lipid

\footnotetext{
Address correspondence to L. Amy Sung, Department of Bioengineering, Jacobs School of Engineering, University of California, La Jolla, San Diego, CA 92093-0412, USA. Electronic mail: amysung@bioeng.ucsd.edu
}

bilayer. ${ }^{3,15,24}$ We previously proposed that a junctional complex (JC) consisting of a short actin protofilament with a $180^{\circ}$ turn, stabilized by two rod-like tropomyosin (TM), capped by an erythrocyte tropomodulin (E-Tmod), and wrapped around by three pairs of long $\alpha \beta$ spectrin $(\mathrm{Sp})$ heterodimers forms the basic repeating unit of the network (Fig. 1b). ${ }^{27}$ Each Sp is associated with the lipid bilayer by a suspension complex (SC), which includes band 3, ankyrin, and protein 4.2. By forming the head-to-head association, Sp connects repeating units and gives the network a topology of "spoked" hexagon (Fig. 1a).

The $\alpha \beta \mathrm{Sp}$ is intertwined and arranged in an antiparallel fashion. ${ }^{9}$ In the head region, the tetramer is formed with the $\mathrm{C}$ terminus of $\beta$ Sp binding to the $\mathrm{N}$ terminus of $\alpha \mathrm{Sp}$, which may dissociate into dimers under shear stress. In the tail region, the $\mathrm{N}$ terminus of $\beta \mathrm{Sp}$ is associated with the $\mathrm{C}$ terminus of $\alpha \mathrm{Sp}$, and binds to the actin protofilament. ${ }^{34} \mathrm{On}$ average, $6 \mathrm{Sp}$ converge to bind to one actin protofilament in a JC. ${ }^{3,15,24}$ There are 20 homologous domains in $\alpha \mathrm{Sp}$ and 16 in $\beta \mathrm{Sp}$, with a 106-residue repeat per domain. ${ }^{26}$ The homologous domain pairing near the tail end is responsible for the nucleation for $\mathrm{Sp}$ dimerization, which is propagated to the head region. The initiation of $\mathrm{Sp}$ dimerization involves complementary electrostatic interactions between paired triple-helical bundles. ${ }^{1}$ Force extension of $\alpha$ or $\beta$ Sp by atomic force microscopy revealed a saw-tooth pattern that arose from the successive unfolding of the homologous domains, each requiring $\sim 30 \mathrm{pN} .^{22}$

We previously proposed a wrap-around model for the $\mathrm{Sp}$ /protofilament junction (Fig. 1d). ${ }^{27}$ In that model, a reinforced protofilament may function as a mechanical axis to anchor three (top, middle, and bottom) pairs of Sp. Each Sp pair may wrap-around 


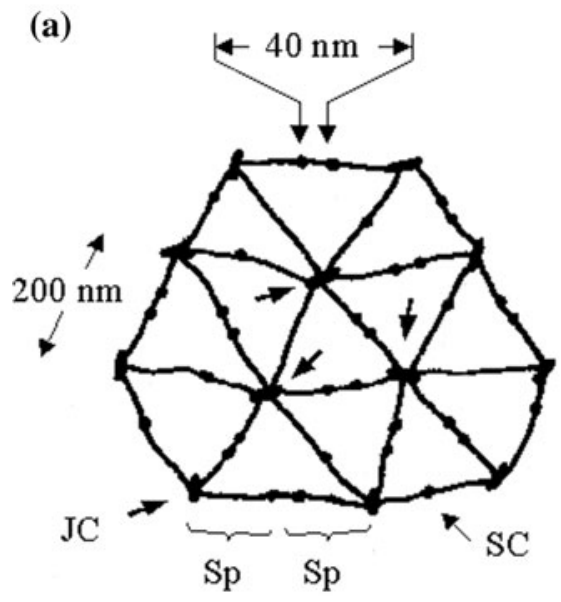

(d)

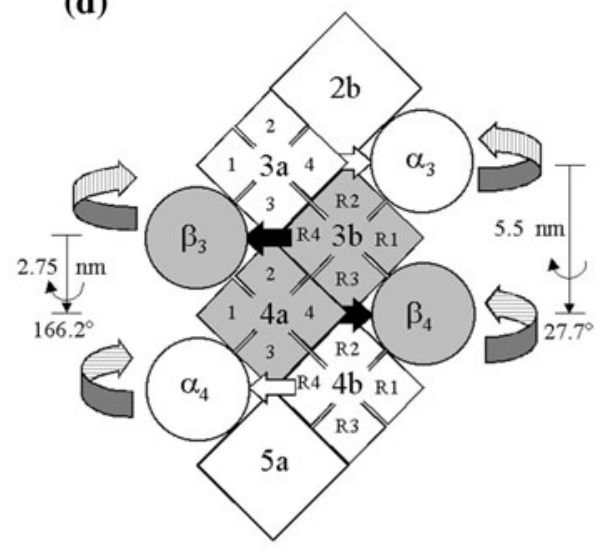

(b)

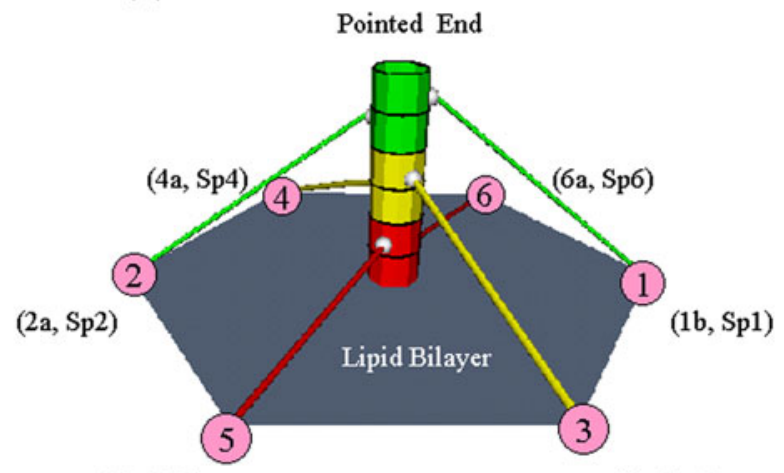

(5b, Sp5)

(3b, Sp3) (c)

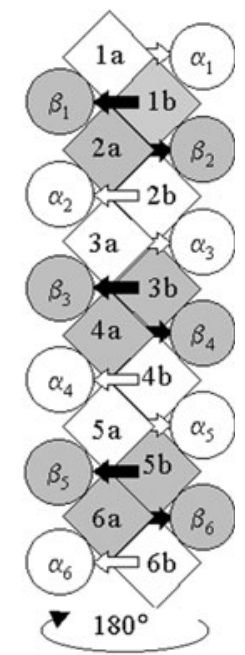

(e)

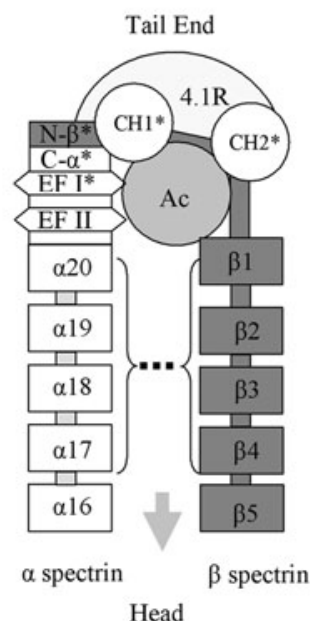

FIGURE 1. Sketches of the erythrocyte membrane skeleton and the wrap-around model from large to small scale. (a) The organization of the skeletal network, showing JC, SC, and Sp; (b) A 3-D view of a JC with a protofilament associated with three pairs of Sp and 6 SC, forming a basic repeating unit. ${ }^{27}$ (The angle between JC and the lipid bilayer is not specified); (c) A protofilament having 12 individual G actin and 6 occupied (filled) binding sites for $\beta \mathrm{Sp}$; (d) An enlarged section of the protofilament bound with the middle pair: Sp3 $(\alpha 3 \beta 3)$ and Sp4 ( $\alpha 4 \beta 4)$. Sp3 wraps around a pair of G actin $(3 a / 3 b)$; Sp4 wraps around the next pair of G actin (4a/4b) in the opposite direction (a-d modified from Ref. 27); (e) The detail sketch of $\alpha$ and $\beta$ spectrin, which split and rejoin to wrap the protofilament. The tail end begins with the non-homologous domains of $\mathrm{N}-\beta$, including $\mathrm{ABD}$ (CH1 and $\mathrm{CH} 2$ ), and that of $\mathrm{C}-\alpha$, including EFs. Cys (*) may form disulfide bonds. The dimer nucleation site spans $\alpha 1-4$ and $\beta 18-21$. This sketch appeared in Ref. 36 without detailed description. The size of linkers (\# of residues): $\mathrm{N}-\beta$ (50), between $\mathrm{CH} 1$ and $\mathrm{CH} 2$ (10), between CH2 and $\beta 1$ (19), between homologous $\beta$ domains (8); C- $\alpha$ (75), between EF1 and EF2 (14), between EFII and $\alpha 20$ (8), and between $\alpha$ homologous domains (8). Molecules and domains are not to scale. The intertwined feature is not shown for clarity purposes.

the protofilament with a wide dihedral angle $\left(\sim 166.2^{\circ}\right)$ and a minimal axial distance $(\sim 2.75 \mathrm{~nm}){ }^{8}$ The $3 \mathrm{Sp}$ pairs may spiral down (right-handed) the protofilament from the pointed end with a dihedral angle of $\sim 55.4^{\circ}$ in between Sp pairs. This first 3-D model of JC may explain why 6 , rather than 12 , Sp bind to a protofilament, and has been used to simulate the 3-D nano-mechanics of a single unit ${ }^{33}$ and multiple units of the membrane skeletal network, ${ }^{5}$ as well as a single unit coupled to the lipid bilayer. ${ }^{36}$ With the support from a number of published biochemical studies, we first illustrated how Sp may wrap-around the actin protofilament by showing the detailed arrangements of actin domains involved (Fig. 1d $)^{27}$ followed by detailed arrangements of $\mathrm{Sp}$ domains involved (Fig. 1e). ${ }^{36}$ In this paper, we detail the wrap-around model in contrast to the point-attachment model generally presented in textbooks or articles. ${ }^{6} \mathrm{We}$ first analyze the interaction between intertwined $\mathrm{Sp}$ and helical protofilament that begins with one split $\mathrm{Sp}$, expands into a pair of $\mathrm{Sp}$, and ends with three pairs of $\mathrm{Sp}$. We then, step-by-step, provide an energy and mechanical basis supporting the wrap-around model, essential for the integrity and elasticity of the erythrocyte membrane skeleton. Lastly, we show how a wrapping-around junction may be converted to a point-attachment junction, leading to the destruction of the network and the death of an erythrocyte. This is 
the novelty of this study advanced from our previous publications.

\section{METHODS}

\section{Strength of Sp Loop}

The binding affinities were converted into free energy, a measure of the reversibility of a reaction, $\Delta G=-R T \ln K_{\mathrm{d}}$, where $R$ is the ideal gas constant $8.3144 \mathrm{~J} / \mathrm{mol} \mathrm{K}, T$ is the absolute reference temperature of the system, and $K_{\mathrm{d}}$ is the binding affinity (dissociation constant) of the interaction measured in the referenced temperature. Thus, the binding energy derived from $K_{\mathrm{d}}$ can be compared at the same temperature among all interactions involving protofilament, $\alpha$ and $\beta \mathrm{Sp}$, and their components.

\section{Potential Energy in Extended Sp}

The energy is the integration of force over the distance of extension. Thus, a small force exerted over a long distance can yield the same energy as that of a large force over a relatively short distance. The nonlinear spring is capable of storing potential energy as a result of force extension. The work done (potential energy expended), $\Delta G$, on force extension is described by its integral:

$$
\Delta G=\int F(l) \mathrm{d} l=\frac{K_{\mathrm{B}} T}{p} \int\left(\frac{1}{4\left(1-\frac{l}{L_{\mathrm{c}}}\right)^{2}}-\frac{1}{4}+\frac{1}{L_{\mathrm{c}}}\right) \mathrm{d} l
$$

Using $K_{\mathrm{B}} T / p=8 \times 10^{-21} \mathrm{~J} / \mathrm{nm}$ and $L_{\mathrm{c}}=144.2 \mathrm{~nm}$, $\Delta G$ can be derived. The value of $K_{\mathrm{B}} T / p$ was derived from the force-extension curve of $\mathrm{Sp}$ in the modified WLC model, ${ }^{22}$ where $K_{\mathrm{B}}$ is the Boltzmann constant, $T$ is the absolute temperature, and $p$ is the persistent length of $\mathrm{Sp}$, corresponding to $0.5 \mathrm{~nm}$.

\section{DOF and 5DOF Simulations}

We have previously described the details of the mathematical models for single unit ${ }^{33}$ and multi-unit simulations. ${ }^{5}$ The protofilament is modeled as a rigid rod with mass $m$ and length $\ell$. The equations of its motion can be described by:

$$
\begin{aligned}
& \ddot{\mathbf{r}}=m^{-1} \mathbf{f}_{\mathrm{r}} \\
& \ddot{\mathbf{b}}=J^{-1}\left(\mathbf{b b}^{T} /\|\mathbf{b}\|^{2}-\mathbf{I}\right) \mathbf{f}_{\mathrm{b}}-(\|\dot{\mathbf{b}}\| /\|\mathbf{b}\|) \mathbf{b}
\end{aligned}
$$

where $\mathbf{r}$ is a vector pointing to the center of mass, $m$, of the protofilament, $\mathbf{b}$ is a unit vector in the direction of the protofilament, and $J$ is the moment of inertia of the rigid protofilament. The generalized forces $\mathbf{f}_{\mathrm{r}}$ and $\mathbf{f}_{\mathrm{b}}$ are calculated as:

$$
\mathbf{f}_{\mathrm{r}}=\sum_{i} \mathbf{f}_{i}, \quad \mathbf{f}_{\mathrm{b}}=\sum_{i} \ell_{i} \mathbf{f}_{i}
$$

where the vector $\mathbf{f}_{i}$ represents a force applied at a node located at distance $\ell_{i}$ measured from $m$. In a typical unit, these forces arise from elongation of $\mathrm{Sp}$, which were modeled as nonlinear elastic elements following the force vs. elongation curve of the WLC model. ${ }^{22}$

We connected rings of units to generate multi-units and subjected networks to an equibiaxial deformation by applying radial force to all peripheral $\mathrm{Sp}$ units (PU) and analyzing the mechanical behavior of all JCs of the network, focusing our attention in the central unit (CU). The simulations are based on the 3-D model of the protein skeletal network alone, not coupled to the lipid bilayer.

6DOF ( 6 degrees of freedom) allows the protofilament to change roll, pitch, and yaw angles, plus $x, y, z$ translations. 5DOF ( 5 degrees of freedom) eliminates the change of the roll angle. Both 6DOF and 5DOF simulations have been conducted using our mathematical models. ${ }^{5,33,36} 6 \mathrm{DOF}$ simulations correspond to point-attachment junction; 5DOF simulations correspond to wrap-around junction. The extension of $\alpha$ and/or $\beta$ Sp wrapping-around the protofilament may minimize the rotational torque in the protofilament during deformation, eliminating the roll motion, which effectively produces a 5DOF system.

\section{RESULTS AND DISCUSSION}

The 3-D model we previously proposed for a $\mathrm{JC}$ in the erythrocyte membrane skeletal network (Fig. 1a) consists of three (top, middle, and bottom) pairs of $\mathrm{Sp}$ wrapping around an actin protofilament (Fig. 1b), clustered in a back-to-back fashion (Figs. 1c, 1d). ${ }^{27}$ Each of the following sections will deal with one specific design feature of the wrap-around model (Fig. 1e), how it was derived, and what consequences it may have. Such a study will help us understand how the membrane skeletal network may sustain mechanical stress and undergo elastic recovery.

\section{Splitting and Rejoining of $S p$}

Sp domains that may wrap-around the protofilament are based on their reported binding affinities. First, the nucleation site for $\alpha \beta$ Sp dimerization occurs between homologous domains ( $\beta 1-4$ and $\alpha 17-20)$ near the tail end, ${ }^{32}$ but not at the terminal non-homologous domains. ${ }^{23}$ This makes the splitting of $\alpha$ and $\beta$ Sp at the non-homologous domains a viable possibility (Fig. 1e). Second, regarding the actin binding domain 
TABLE 1. Binding affinity and converted free energy in interactions involving actin and spectrin.

\begin{tabular}{|c|c|c|c|c|c|}
\hline Interaction & $K_{\mathrm{d}}(\mu \mathrm{M})$ & Ref. & $T\left({ }^{\circ} \mathrm{C}\right)$ & $\Delta G(\mathrm{~kJ} / \mathrm{mol})$ & $\begin{array}{c}\Delta G \text { at } 23^{\circ} \mathrm{C} \\
(\mathrm{kJ} / \mathrm{mol})\end{array}$ \\
\hline$(\mathrm{CH} 1+\mathrm{CH} 2) /$ actin & 26 & 14 & 24 & 26 & 26 \\
\hline$(\mathrm{CH} 1+\mathrm{CH} 2+\beta 1) /$ actin & 6 & 14 & 24 & 30 & 30 \\
\hline$\left(\alpha 19+\alpha 20+\mathrm{CH}_{1}+\mathrm{CH} 2+\beta 1\right) /$ actin & 2.5 & 20 & Room $T(20)$ & 31 & 32 \\
\hline$(\beta+$ actin $) /$ protein $4.1 \mathrm{R}$ & 0.10 & 31 & 4 & 37 & 40 \\
\hline Sp/protein 4.1R & 0.011 & 20 & 24 & 45 & 45 \\
\hline$(\alpha 17-20) /(\beta 1-4)$ (nucleation) & $0.01-0.015$ & 32 & 4 & 42 & 45 \\
\hline Sp/Sp (head-to-head) & 1.7 & 4 & 35 & 34 & 33 \\
\hline
\end{tabular}

(ABD) on $\beta \mathrm{Sp}$ : Among the non-homologous domains (Table 1), the calponin homology domain 1 (CH1) binds to F-actin at $10 \%$ affinity of $\mathrm{CH} 1$ plus $\mathrm{CH} 2,{ }^{11}$ while $\mathrm{CH} 2$ alone does not. Adding $\beta 1$ domain increased the affinity by fourfold, but further inclusion of $\beta 2$ domain did not. ${ }^{13}$ Therefore, we position $\mathrm{CH} 1$, $\mathrm{CH} 2$ and $\beta 1$ around the protofilament, with $\mathrm{CH} 1$ binding to F-actin and the other two domains anchored in the clefts (Figs. 1d, 1e), reducing the stress on $\mathrm{CH} 1$.

The rejoining of $\alpha$ and $\beta$ Sp behind protofilament and "nailed" by protein $4.1 \mathrm{R}$ are based on: (A) The inter-chain binding between $\alpha$ and $\beta$ Sp involves terminal non-homologous domains. ${ }^{34}$ (B) Some Cys residues near the tail end are reactive and may form disulfide bonds. ${ }^{9}$ (C) Protein 4.1R stabilizes the binding between $\beta \mathrm{Sp}$ and $\mathrm{F}$-actin (Table 1), with a split $\beta$ Sp binding site (binding to $\mathrm{CH} 1$ and $\mathrm{CH} 2$, respectively) and an actin binding site in between (Fig. 1e). ${ }^{6,10}$ While covalent bonds would complete the loop with a greater strength, protein $4.1 \mathrm{R}$ would hold $\mathrm{Sp} /$ actin together and restrict Sp spinning around the filament. ${ }^{27}$

\section{Actin Inside Sp Loop}

Some $\mathrm{CH}$ domains switch between open and closed conformations; ${ }^{28}$ some EF domains have open and close configurations. ${ }^{29}$ The $\mathrm{CH}$ and EF domains and linkers (Fig. 1e) around the protofilament may allow Sp to redirect without rolling the protofilament (see "Methods" section). Previous investigations on $\mathrm{Ca}^{2+} /$ calmodulin regulations indicated that opening $\mathrm{EF}$ domains (containing a helix-loop-helix motif that usually binds $\mathrm{Ca}^{2+}$ ) decreases the binding affinity of the $\mathrm{ABD}$ of a neighboring molecule that is in direct contact. ${ }^{28}$ However, a minimum Sp construct, ${ }^{21}$ containing EF domains of $\alpha \mathrm{Sp}$ and $\mathrm{CH}$ domains (ABD) of $\beta \mathrm{Sp}$, was found unaffected by up to $100 \mu \mathrm{m} \mathrm{Ca}{ }^{2+}$. This finding is in agreement with the wrap-around model: $\mathrm{EF}$ and $\mathrm{CH}$ domains are not in direct contact (actin is in between).

The circumference of a protofilament $(9 \mathrm{~nm}$ in diameter) is $\sim 28 \mathrm{~nm}$. Approximately $150^{23}$ and $272^{35}$ residues in the non-homologous region of $\alpha$ and $\beta \mathrm{Sp}$, respectively, would be more than enough to cover the circumference. The dimer nucleation site would be on the other side of the protofilament (Fig. 1e). Transmission electron microscopy revealed a loop structure (average diameter of $24 \mathrm{~nm}$ ) at one end of Sp dimers in vitro (average contour length of $103 \mathrm{~nm}$ ) (see Fig. 8 in Ref. 25), providing direct physical evidence supporting the wrap-around model (Fig. 2b).

\section{Sp Loop is Not Pre-formed}

It would be difficult to place six pre-formed Sp loops around one protofilament. It has been reported that $\beta$ $\mathrm{Sp}$ is synthesized and assembled into the membrane skeleton early; thereafter, $\alpha \mathrm{Sp}$ is produced, in threefold molar excess to $\beta \mathrm{Sp}$ and associates with $\beta \mathrm{Sp}$ to $95 \%$ extent. ${ }^{18}$ Since $\beta$ Sp binding to a protofilament takes place strategically one step before closing the loop by $\alpha \mathrm{Sp}$, this sequence of event in vivo solves the problem of having six loops crossing over one another during the construction of a JC.

\section{$J C$ is Not a Pulley}

Topologically split $\alpha$ and $\beta$ Sp wrapping around a protofilament (Fig. 1e) resembles a rope around a pulley; however, their designs and functions are different. In a pulley, the horizontal force applied to one side of the rope (sliding) is to generate rotational force or torque, causing the mechanical axis to rotate. In JC, the protofilament has a limited degree of torsional flexibility as previously stated, ${ }^{30}$ and extensive torque generated by sliding $6 \mathrm{Sp}$ in different ways would be detrimental to the protofilament. Fortunately, intertwining between $\alpha$ and $\beta$ Sp (to avoid sliding) and $\mathrm{Sp}$ swinging (not spinning) around the axis may minimize the torque. (See Figs. 1e, 2b and sections below.)

\section{Network Integrity}

Table 1 shows $\Delta G$ at $23{ }^{\circ} \mathrm{C}$ of every joint involved in the $\mathrm{Sp} \mathrm{loop},{ }^{4,14,20,31}$ deriving from the binding 

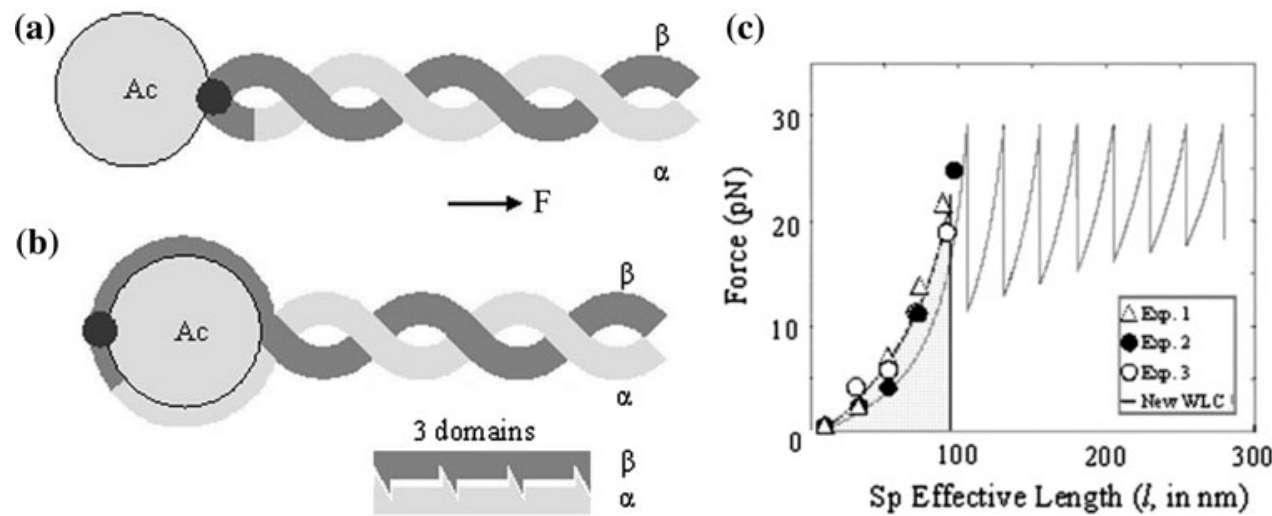

FIGURE 2. Point-attachment vs. wrap-around. (a) In a point-attachment junction, the ABD binds to a single point (solid circle) on the front side of the protofilament and force applied through Sp directly affects the binding. The unfolding (and open/close configurations) of domains would be the primary protective measure. (b) In a wrap-around junction, the ABD binds to the back side of the protofilament. $\alpha$ and $\beta$ spectrin are arranged in an anti-parallel orientation and resists sliding through steric hindrance which is enhanced by intertwining. The greater the extension, the tighter the intercalating would be. (c) Modified force-extension curve of single stranded spectrin. ${ }^{22}$ The saw-tooth pattern represents successive unfolding of single spectrin repeats. The pointattachment junction features an irreversible drop (a vertical line) at the threshold at the point of detachment between Sp and protofilament. The lower panel shows 3 domains in each pitch.

affinities as a measure of the reversibility of a reaction (see "Methods" section). The highest energy level occurs in nucleation and in the $\mathrm{Sp} /$ protein $4.1 \mathrm{R}$ interaction (both without $\mathrm{F}$-actin), suggesting that the strength holding $\alpha$ and $\beta$ Sp together on the opposite sides of the protofilament is about equal (Fig. 1e). This is a good mechanical design since the loop bears no particular weak point. This further implies that in the event ABD is dissociated from the protofilament, the Sp loop may still remain intact around the protofilament, making their re-association easier. Furthermore, the energy holding the Sp heads together in a tetramer is weaker than that in the tail around the protofilament, suggesting that "breathing" (i.e., an action of Sp tetramer dissociation and reformation) is more likely to occur than breaking the Sp loop around the protofilament. It is easier for the network to repair during breathing, since Sp heads are all "suspended" by SC near the lipid bilayer, facilitating their re-association; new Sp loops, on the other hand, are harder to reform since the environment favoring the loop formation no longer exists (e.g., threefold excess of $\alpha \mathrm{Sp}$ ) once a reticulocyte matures into an erythrocyte.

\section{Point-Attachment vs. Wrap-Around}

From a mechanical point of view, a point-attachment junction has several disadvantages over a wrap-around junction. Firstly, the point-attachment junction (Fig. 2a) places the integrity of a JC on primarily the strength of ABD. As Sp attaches the protofilament through a point or minimal area, the force per unit of area acting to separate the interaction is much larger than that in the wrap-around junction, where the contact area is maximized (Fig. 2b). Secondly, the tension of $\mathrm{Sp}$ can serve directly and completely to weaken the actin binding, leading to their separation (in the same direction as the tension) if ABD is in front of the protofilament, whereas a $\mathrm{Sp}$ tension would enhance the association (compression, not separation) if ABD is on the back of the protofilament. Thirdly, as $\mathrm{Sp}$ swings around the protofilament, the pointattachment junction requires that $\mathrm{Sp}$ changes its angle relative to the protofilament at $\mathrm{ABD}$, which may cause conformational changes and weaken their binding and result in detachment, whereas the wrap-around junction would not impose changes of angle at ABD as previously described. Lastly, once $\mathrm{Sp}$ physically detaches from the protofilament, large entropy is gained, making it unfavorable to restore the original configuration in the point-attachment junction, whereas an intact Sp loop remaining around a protofilament would make their re-association easier. Furthermore, in the wrap-around junction, the nucleation site may prevent $\alpha$ and $\beta$ Sp separation in front of the protofilament during $\mathrm{Sp}$ extension and/or to facilitate nucleation again when $\mathrm{Sp}$ relaxes. In the pointattachment junction, the nucleation site would not exert any function since it does not participate in this event. In the wrap-around junction, the stress at ABD may approach zero, if (1) the plane of ABD is perpendicular to the force applied; (2) frictions between Sp and F-actin are high; and/or (3) the energy is used to deform domains or overcome frictions/anchorages preceding $\mathrm{CH} 1$ (see Fig. 1e). Our wrap-around model is based on the geometry of the $\mathrm{JC}$ and the molecular structures and stoichiometry of its key components. Thus it was logically derived and predicts results. 
However, innovative experiments will be required to provide evidence for these two types of junctions.

\section{Intertwined Sp with Anti-Parallel Domains}

Sliding force between $\alpha$ and $\beta \mathrm{Sp}$ or friction is important because of their anti-parallel arrangement. The friction occurs, for example, when one is stationary while the other is subjected to force and the two are intertwined with their surfaces interlocked. On average, there are 5 turns per $\alpha$ or $\beta$ Sp and 3 homologous domains per turn (Fig. 2b, lower panel). ${ }^{17}$ Intertwining $\alpha$ and $\beta$ Sp may bring their domains closer to each other especially during extension. Sliding between $\alpha$ and $\beta \mathrm{Sp}$ may rotate the protofilament like in a pulley; therefore, intertwining $\alpha$ and $\beta \mathrm{Sp}$ in an anti-parallel fashion may decrease the likelihood of sliding against each other, reducing the rolling of the protofilament. If $\alpha$ and $\beta$ Sp are completely registered without any weak point to unfold, Sp may behave like one strong rope.

\section{Before Sp Unfolding}

The stored potential energy of an extended $\alpha$ or $\beta \mathrm{Sp}$ can be calculated from the area under the forceextension curve (Fig. 2c). Using the modified WLC model (see "Methods" section) and $21 \mathrm{pN}$ as the average threshold value of forces for the first unfolding, the maximum potential energy $(\Delta G)$ stored in $\alpha$ or $\beta$ Sp corresponds to $632 \times 10^{-21} \mathrm{~J}$ or $381 \mathrm{~kJ} / \mathrm{mol}$. This suggests that when $\alpha$ or $\beta \mathrm{Sp}$ is extended $95.7 \mathrm{~nm}$ beyond its original distance between protofilament and SC $(\lambda=3.191)$, this level of energy can be stored without unfolding any $\mathrm{Sp}$ domain. There are numerous interactions within the three helices in each domain and a large number of domains exist in $\alpha$ or $\beta \mathrm{Sp}$, so potential energy can gradually accumulate among all domains and eventually reach such a large value.

For a single Sp domain, the energy level that can store before unfolding is $\sim 25 \mathrm{~kJ} / \mathrm{mol}(\sim 381 \mathrm{~kJ} / \mathrm{mol}$ divided by 15 , the number of domains between $\mathrm{JC}$ and $\mathrm{SC}$ on $\beta \mathrm{Sp}$ ). This is generally less energy than needed to disrupt the interactions between $\mathrm{Sp}$ and protofilament $(\sim 32 \mathrm{~kJ} / \mathrm{mol})$ or between Sp heads $(\sim 33 \mathrm{~kJ} / \mathrm{mol})$ (Table 1). Therefore, if stress is applied (horizontally) to the network, and if $\alpha$ and $\beta \mathrm{Sp}$ are not registered, single Sp domain unfolding may occur before disruption of any other interactions, including "breathing" of Sp (see Table 1).

If $\alpha$ and $\beta \mathrm{Sp}$ are completely registered the maximal total energy stored in the intertwined rope would be approximately twice of $381 \mathrm{~kJ} / \mathrm{mol}$. In that case, the rope can be subjected to a greater stress without unfolding or breaking (a covalent bond is $\sim 210$ $420 \mathrm{~kJ} / \mathrm{mol}$ ), and "breathing" of Sp may occur. If $\alpha$ and $\beta$ Sp are partially registered, simultaneous unfolding of domains from both strands are possible. ${ }^{13}$

\section{Sp in Tension}

Our 3-D model for a JC predicts that $6 \mathrm{Sp}$ are clustered into three pairs, in a back-to-back fashion, with a widest possible dihedral angle $\left(166.2^{\circ}\right)$ and a minimal axial distance $(2.75 \mathrm{~nm})$ within the constrain of $\mathrm{G}$ actin in the filament (Fig. 1d). ${ }^{27}$ While the wide dihedral angle tends to balance the forces (Figs. 3a, 3d, 1d), a minimal axial distance tends to reduce the bending moment (Figs. 3d, 1d). Consequently, the protofilament is unlikely to flip globally or bend locally by any given pair of $\mathrm{Sp}$ when they are subjected to tension.

Figure 3 illustrates a small portion of the network under equibiaxial extension (panel $\mathrm{A}$ ), a $\mathrm{Sp}$ tetramer formed head-to-head between two JC (panel B), and

\section{(a)}

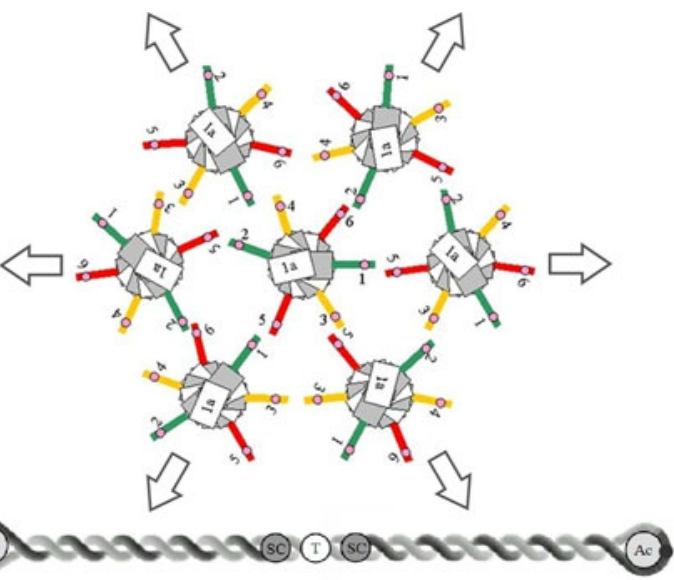

(c)
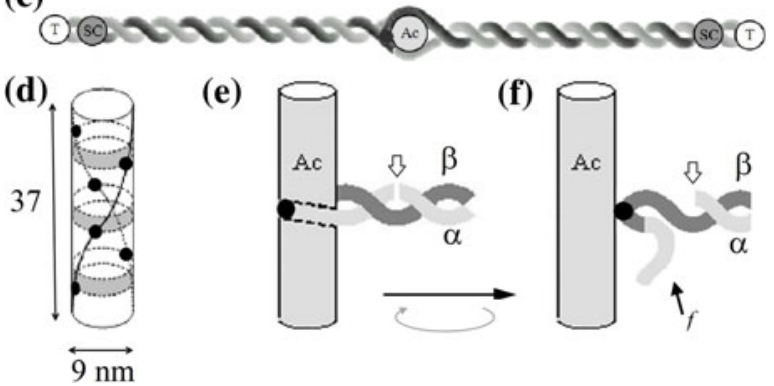

(e)

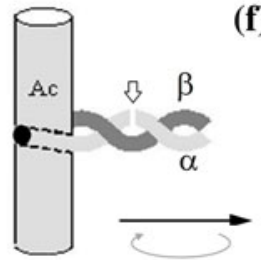

(f)

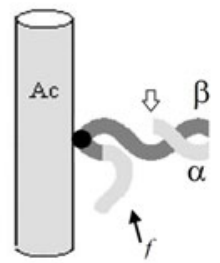

FIGURE 3. The conversion of a wrap-around junction to a point-attachment junction. (a) A two layer network (consisting of $1 \mathrm{CU}$ and $6 \mathrm{PU}$ ) under equibiaxial extension. (b) A pair of SC at the center of a Sp tetramer. ${ }^{4}$ (c) A JC wrapped around by a pair of Sp (see Fig. 1d). (d) Side view of a JC, showing 6 occupied ABD (solid circles), 3 in each opposing helix, spiral from one end to the other. (e) One ABD in a wrap-around junction before a single strand cleavage (e.g., on $\alpha$ spectrin) (open arrow). (f) The cleavage may free the $f$ segment from the protofilament and roll the protofilament during extension, converting a wrap-around junction to a point-attachment junction. AC: actin protofilament. T: Sp tetramerization site. $f$ segment: a segment of single stranded spectrin between ABD and the cleavage site (may consist only $\beta$ spectrin or a combination of $\alpha$ and $\beta$ spectrin connected at the tail end). 
a JC wrapped around by a pair of $\mathrm{Sp}$ in opposite direction (panel $\mathrm{C}$ ). Three pairs of $\mathrm{ABD}$ along the protofilament (panel D), allow a JC to undergo equibiaxial extension (with all $3 \mathrm{Sp}$ pairs in tension) or anisotropic extension (when only one or two Sp pairs in tension). The side view of a protofilament wrapped around by a $\mathrm{Sp}$ is in panel $\mathrm{E}$. The potential energy stored in the extended Sp may be used to shorten Sp and help restoring the membrane skeleton to the original state once the external force is removed, providing the molecular basis for the elasticity of the erythrocyte membrane skeleton. However, if one or two Sp pairs are severed, the erythrocyte may only be able to restore in the direction where Sp remains intact, leading to the formation of ovalocytes or elliptocytes.

\section{Strain in Protofilament}

There is $180^{\circ}$ turn per protofilament of $37 \mathrm{~nm}$. Each of the two spiral grooves (Fig. 3d) may be occupied by a double-helical TM molecule of $\sim 35 \mathrm{~nm}$ (in erythrocytes) that reinforces the integrity of the protofilament. ${ }^{27}$ The $\mathrm{G}$ actin subunits are known to have some torsional freedom to rotate against one another (maximal of $\sim 10^{\circ}$ ),${ }^{30}$ but during an axial extension the $180^{\circ}$ spiral grooves may not be straightened purely by the torsional freedom. The force required for such torsional changes is in the order of $\mathrm{nN}$, so when stresses are applied to the membrane skeletal network during erythrocyte deformation (if events such as swinging, unfolding, and "breathing" of $\mathrm{Sp}$ are allowed) they would have occurred before the strain of the protofilament and TM. Should a protofilament be fractured horizontally, TMs positioned vertically one in each of the spiral grooves (like two face-to-face brackets) may help to retain $G$ actin subunits and restore the conformation of the helical protofilament.

\section{Converting Wrap-Around to Point-Attachment}

If there is a single strand severance (e.g., digestion by calcium-activated enzymatic cleavage ${ }^{19}$ ) anywhere along $\alpha$ or $\beta \mathrm{Sp}$, a wrap-around junction (Fig. 3e) will be converted into a point-attachment junction (Fig. 3f). In that case, a Sp extension may bring the $\mathrm{ABD}$ of $\beta \mathrm{Sp}$ to the front (from the back) by rolling the protofilament during extension. It has been reported that shearing of erythrocytes induces calcium influx. ${ }^{12}$ Therefore, erythrocyte deformation in a small blood vessel may induce single strand severance (or double strand severance if the digestion continues). With a point-attachment junction, a $\mathrm{Sp}$ redirection tends to roll the protofilament, and a Sp extension tends to break the $\mathrm{Sp} /$ protofilament binding as described in previous sections.

\section{DOF and 5DOF Simulations}

We have previously simulated the 3-D nanomechanics of a single unit or multiple units of the membrane skeleton using a 6DOF model (for pointattachment), or 5DOF model (without roll movement, for wrap-around). These simulations predict the attitude of a protofilament (in pitch, yaw, and roll angles) and the tension of each $\mathrm{Sp}$ (in $\mathrm{pN}$ ) in response to 2.5$30 \mathrm{pN}$, corresponding to equibiaxial extension ratios of up to 3.4, which are within the physiological range.

Our 6DOF simulations for a single unit (when the freedom of SC is restricted and a force is applied representing its interaction with the lipid bilayer) using both the dynamic relaxation method ${ }^{33}$ and the hybrid model $^{36}$ revealed a "bifurcation" of the equilibrium state during anisotropic (or shear) deformation, while the $5 \mathrm{DOF}$ simulation rarely has done so. ${ }^{36}$ At the "bifurcation", the yaw angle of the protofilament changed drastically (e.g., $72^{\circ}$ to $-72^{\circ}$ ) within a short time is often accompanied by an abrupt increase of pitch angle (up to $80^{\circ}$ ), indicating a "bow up" motion. In our multi-unit network simulations (when the freedom of SC is not restricted except at the periphery), ${ }^{5}$ the 5DOF network under equibiaxial extension reached equilibrium (for example, $\lambda=1.3$ in Fig. 4, upper panels) with a pitch angle of $\sim 0^{\circ}$, even without any applied force representing its interaction with the lipid bilayer. On the other hand, the 6DOF network did not reach equilibrium and was accompanied by numerous "bow up" motions of protofilaments (bottom panels). These series of simulations in the past illustrate the importance of $\mathrm{Sp}$ /protofilament junctions in the 3-D nano-mechanics of the membrane skeleton.

These simulations have lead to our current hypothesis that if a protofilament "bows up", or tumbles, it may cause a local disturbance or flipping of the overlaying lipid bilayer. This may provide a mechanical basis for flipping negatively charged phosphotidylserine from inner leaflet to outer leaflet. It has been reported that this well characterized "eat-me" signal marks stressed (e.g., aged or diseased) erythrocytes for sequestration. ${ }^{2,16}$ Thus, in this study, we propose a new mechanism for bringing about such critical signals: converting a wrap-around junction to a point-attachment junction by a cleavage or injury of the membrane skeletal network. To test, we subjected erythrocytes to shear stress that induces calcium influx, ${ }^{12}$ a condition known to activate enzymatic digestion of Sp. ${ }^{19}$ Indeed, these treatments externalized phosphotidylserine and enhanced erythrophagocytosis (unpublished results, Peng and Sung). Even though these results are not direct evidence, and may involve more than one mechanism, they are clearly in agreement with our model predictions. 


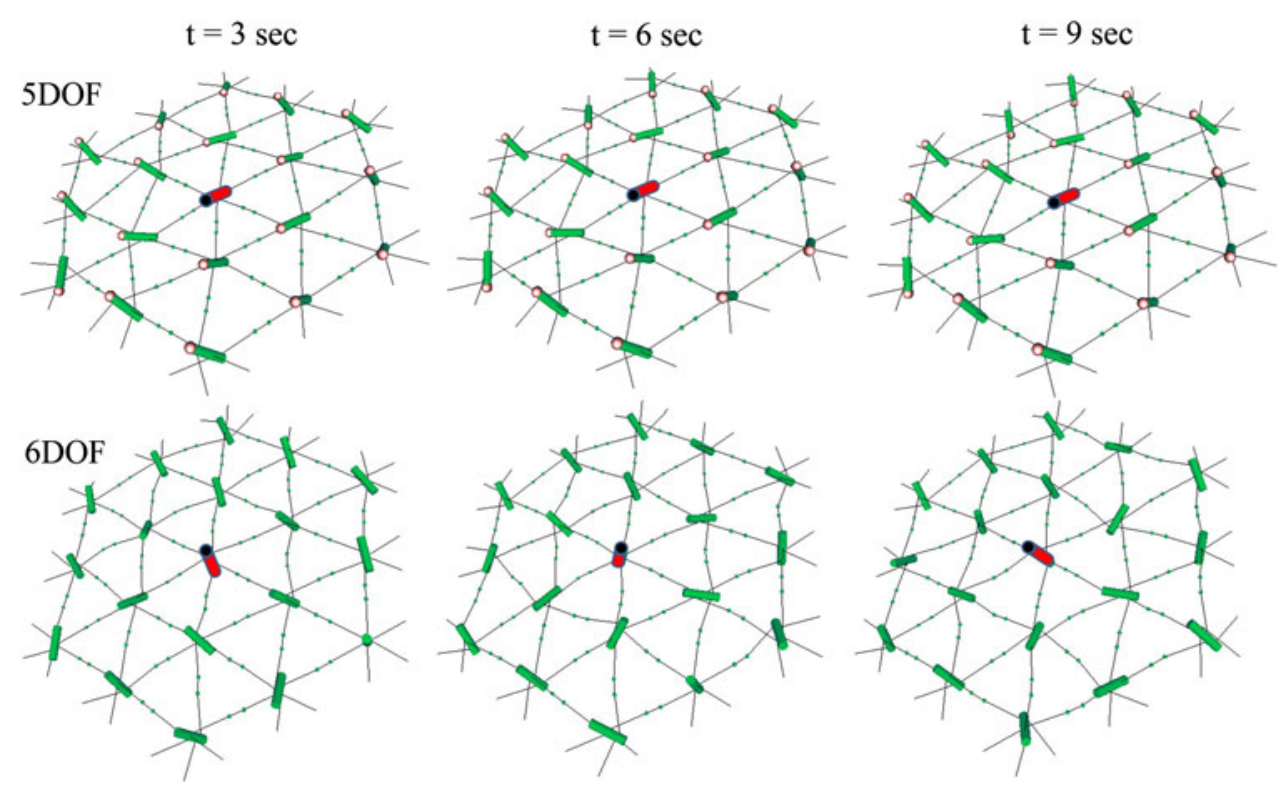

FIGURE 4. Snap shots of a membrane skeletal network in 5DOF and 6DOF simulations during equibiaxial extension ( $\lambda=1.3)$. Upper panels are 5DOF simulations (wrap-around junction) of a network with three layers at 3, 6, and 9 s; lower panels are their corresponding 6DOF simulations (point-attachment junction) based on Ref. 5. Note the "bow up" motion of the protofilament in CU (in red) in the 6DOF simulation. The point end of the protofilament is indicated by a black sphere (E-Tmod). See supplementary data for more information and complete animations.

Simulations of the nano-mechanics of membrane skeletal network based on the wrap-around model also provide new understanding into why deformations of erythrocytes in small capillaries may facilitate the transport of $\mathrm{O}_{2}$ and $\mathrm{CO}_{2}$ across the membrane. ${ }^{5} \mathrm{We}$ are in the process of simulating the networks under anisotropic deformation and during the transition between equibiaxial and shear deformations. We believe such results in the nano-scale and at the molecular level would indeed bring further insight into the microscopic deformation behaviors and functions such as the tank-treading when erythrocytes are subjected to viscometric flow. ${ }^{7}$

\section{SUMMARY}

We detail the proposed wrap-around model for a JC in the erythrocyte membrane skeleton. We describe how components of $\alpha$ and $\beta$ Sp may split and rejoin around an actin protofilament and the advantages from the energy and mechanical points of view for the integrity the network. The wrap-around model minimizes the stress between ABD and protofilament as compared to the point-attachment model. In the wraparound model, Sp and protofilament remain topologically connected, even physically separated, making their re-association more likely. Wrapping and swinging $\mathrm{Sp}$ around the protofilament, pairing $\mathrm{Sp}$ in a backto-back fashion, and spanning three pairs of $\mathrm{Sp} 360^{\circ}$ around the protofilament from top to bottom provide a series of protective mechanisms for the integrity and stability of the membrane skeletal network. Severing $\alpha$ or $\beta$ Sp may convert the $\mathrm{Sp} /$ protofilament interaction from a wrap-around junction to a pointattachment junction, leading to tumbling of $\mathrm{JC}$ along with the "flipping" of the membrane lipid bilayer, marking erythrocytes for sequestration through erythrophagocytosis.

\section{ELECTRONIC SUPPLEMENTARY MATERIAL}

The online version of this article (doi:10.1007/ s10439-011-0293-6) contains supplementary material, which is available to authorized users.

\section{ACKNOWLEDGMENTS}

This work was supported by NIH Research Grant P01HL-43026-6 and Paul Sche was a postdoctoral fellow supported by NIH training grant T32 HL007089.

\section{OPEN ACCESS}

This article is distributed under the terms of the Creative Commons Attribution Noncommercial License which permits any noncommercial use, distribution, and reproduction in any medium, provided the original author(s) and source are credited. 


\section{REFERENCES}

${ }^{1}$ Begg, G. E., S. L. Harper, M. B. Morris, and D. W. Speicher. Initiation of spectrin dimerization involves complementary electrostatic interactions between paired triple-helical bundles. J. Biol. Chem. 275(5):3279-3287, 2000.

${ }^{2}$ Bratosin, D., J. Mazurier, J. P. Tissier, C. Slomianny, J. Estaquier, F. Russo-Marie, J. J. Huart, J. M. Freyssinet, D. Aminoff, J. C. Ameisen, et al. Molecular mechanisms of erythrophagocytosis. Characterization of the senescent erythrocytes that are phagocytized by macrophages. $C$. $R$. Acad. Sci. III 320(10):811-818, 1997.

${ }^{3}$ Byers, T. J., and D. Branton. Visualization of the protein associations in the erythrocyte membrane skeleton. Proc. Natl Acad. Sci. USA 82(18):6153-6157, 1985.

${ }^{4}$ Cherry, L., N. Menhart, and L. W. Fung. Interactions of the alpha-spectrin $\mathrm{N}$-terminal region with beta-spectrin Implications for the spectrin tetramerization reaction. J. Biol. Chem. 274(4):2077-2084, 1999.

${ }^{5}$ de Oliveira, M., C. Vera, P. Valdez, Y. Sharma, R. Skelton, and L. A. Sung. Nanomechanics of multiple units in the erythrocyte membrane skeletal network. Ann. Biomed. Eng. 38(9):2956-2967, 2010.

${ }^{6}$ Discher, D. E., R. Winardi, P. O. Schischmanoff, M. Parra, J. G. Conboy, and N. Mohandas. Mechanochemistry of protein 4.1's spectrin-actin-binding domain: ternary complex interactions, membrane binding, network integration, structural strengthening. J. Cell Biol. 130(4):897-907, 1995.

${ }^{7}$ Fischer, T. M., M. Stohr-Lissen, and H. Schmid-Schonbein. The red cell as a fluid droplet: tank tread-like motion of the human erythrocyte membrane in shear flow. Science 202(4370):894-896, 1978.

${ }^{8}$ Fujii, T., A. H. Iwane, T. Yanagida, and K. Namba. Direct visualization of secondary structures of F-actin by electron cryomicroscopy. Nature 467(7316):724-728, 2010.

${ }^{9}$ Harper, S. L., G. E. Begg, and D. W. Speicher. Role of terminal nonhomologous domains in initiation of human red cell spectrin dimerization. Biochemistry 40(33):99359943, 2001.

${ }^{10}$ Horne, W. C., S. C. Huang, P. S. Becker, T. K. Tang, and E. J. Benz, Jr. Tissue-specific alternative splicing of protein 4.1 inserts an exon necessary for formation of the ternary complex with erythrocyte spectrin and F-actin. Blood 82(8):2558-2563, 1993.

${ }^{11}$ Keep, N. H., F. L. Norwood, C. A. Moores, S. J. Winder, and J. Kendrick-Jones. The 2.0 A structure of the second calponin homology domain from the actin-binding region of the dystrophin homologue utrophin. J. Mol. Biol. 285(3):1257-1264, 1999.

${ }^{12}$ Larsen, F. L., S. Katz, B. D. Roufogalis, and D. E. Brooks. Physiological shear stresses enhance the $\mathrm{Ca} 2+$ permeability of human erythrocytes. Nature 294(5842):667-668, 1981.

${ }^{13}$ Law, R., S. Harper, D. W. Speicher, and D. E. Discher. Influence of lateral association on forced unfolding of antiparallel spectrin heterodimers. J. Biol. Chem. 279(16): 16410-16416, 2004.

${ }^{14} \mathrm{Li}, \mathrm{X}$., and V. Bennett. Identification of the spectrin subunit and domains required for formation of spectrin/ adducin/actin complexes. J. Biol. Chem. 271(26):1569515702,1996

${ }^{15}$ Liu, S. C., L. H. Derick, and J. Palek. Visualization of the hexagonal lattice in the erythrocyte membrane skeleton. J. Cell. Biol. 104(3):527-536, 1987.
${ }^{16}$ Mandal, D., A. Mazumder, P. Das, M. Kundu, and J. Basu. Fas-, caspase 8-, and caspase 3-dependent signaling regulates the activity of the aminophospholipid translocase and phosphatidylserine externalization in human erythrocytes. J. Biol. Chem. 280(47):39460-39467, 2005.

${ }^{17}$ McGough, A. M., and R. Josephs. On the structure of erythrocyte spectrin in partially expanded membrane skeletons. Proc. Natl Acad. Sci. USA 87(13):5208-5212, 1990.

${ }^{18}$ Moon, R. T., and E. Lazarides. beta-Spectrin limits alphaspectrin assembly on membranes following synthesis in a chicken erythroid cell lysate. Nature 305(5929):62-65, 1983.

${ }^{19}$ Pant, H. C., M. Virmani, and P. E. Gallant. Calciuminduced proteolysis of spectrin and band 3 protein in rat erythrocyte membranes. Biochem. Biophys. Res. Commun. 117(2):372-377, 1983

${ }^{20}$ Podgorski, A., and D. Elbaum. Properties of red cell membrane proteins: mechanism of spectrin and band 4.1 interaction. Biochemistry 24(27):7871-7876, 1985.

${ }^{21}$ Raae, A. J., S. Banuelos, J. Ylanne, T. Olausson, K. N. Goldie, T. Wendt, A. Hoenger, and M. Saraste. Actin binding of a minispectrin. Biochim. Biophys. Acta. 1646(1-2): 67-76, 2003.

${ }^{22}$ Rief, M., J. Pascual, M. Saraste, and H. E. Gaub. Single molecule force spectroscopy of spectrin repeats: low unfolding forces in helix bundles. J. Mol. Biol. 286(2):553-561, 1999.

${ }^{23}$ Sahr, K. E., P. Laurila, L. Kotula, A. L. Scarpa, E. Coupal, T. L. Leto, A. J. Linnenbach, J. C. Winkelmann, D. W. Speicher, V. T. Marchesi, et al. The complete cDNA and polypeptide sequences of human erythroid alpha-spectrin. J. Biol. Chem. 265(8):4434-4443, 1990.

${ }^{24}$ Shen, B. W., R. Josephs, and T. L. Steck. Ultrastructure of the intact skeleton of the human erythrocyte membrane. J. Cell Biol. 102(3):997-1006, 1986.

${ }^{25}$ Shotton, D. M., B. E. Burke, and D. Branton. The molecular structure of human erythrocyte spectrin. Biophysical and electron microscopic studies. J. Mol. Biol. 131(2):303-329, 1979

${ }^{26}$ Speicher, D. W., and V. T. Marchesi. Erythrocyte spectrin is comprised of many homologous triple helical segments. Nature 311(5982):177-180, 1984

${ }^{27}$ Sung, L. A., and C. Vera. Protofilament and hexagon: a three-dimensional mechanical model for the junctional complex in the erythrocyte membrane skeleton. Ann. Biomed. Eng. 31(11):1314-1326, 2003.

${ }^{28}$ Tang, J., D. W. Taylor, and K. A. Taylor. The three-dimensional structure of alpha-actinin obtained by cryoelectron microscopy suggests a model for $\mathrm{Ca}(2+)$ dependent actin binding. J. Mol. Biol. 310(4):845-858, 2001.

${ }^{29}$ Trave, G., P. J. Lacombe, M. Pfuhl, M. Saraste, and A. Pastore. Molecular mechanism of the calcium-induced conformational change in the spectrin EF-hands. EMBO J. 14(20):4922-4931, 1995.

${ }^{30}$ Tsuda, Y., H. Yasutake, A. Ishijima, and T. Yanagida. Torsional rigidity of single actin filaments and actin-actin bond breaking force under torsion measured directly by in vitro micromanipulation. Proc. Natl Acad. Sci. USA 93(23):12937-12942, 1996

${ }^{31}$ Tyler, J. M., B. N. Reinhardt, and D. Branton. Associations of erythrocyte membrane proteins. Binding of purified bands 2.1 and 4.1 to spectrin. J. Biol. Chem. 255(14):7034-7039, 1980.

${ }^{32}$ Ursitti, J. A., L. Kotula, T. M. DeSilva, P. J. Curtis, and D. W. Speicher. Mapping the human erythrocyte betaspectrin dimer initiation site using recombinant peptides 
and correlation of its phasing with the alpha-actinin dimer site. J. Biol. Chem. 271(12):6636-6644, 1996.

${ }^{33}$ Vera, C., R. Skelton, F. Bossens, and L. A. Sung. 3-d nanomechanics of an erythrocyte junctional complex in equibiaxial and anisotropic deformations. Ann. Biomed. Eng. 33(10):1387-1404, 2005.

${ }^{34}$ Viel, A., and D. Branton. Interchain binding at the tail end of the Drosophila spectrin molecule. Proc. Natl Acad. Sci. USA 91(23):10839-10843, 1994.
${ }^{35}$ Winkelmann, J. C., J. G. Chang, W. T. Tse, A. L. Scarpa, V. T. Marchesi, and B. G. Forget. Full-length sequence of the cDNA for human erythroid beta-spectrin. J. Biol. Chem. 265(20):11827-11832, 1990.

${ }^{36}$ Zhu, Q., C. Vera, R. J. Asaro, P. Sche, and L. A. Sung. A hybrid model for erythrocyte membrane: a single unit of protein network coupled with lipid bilayer. Biophys. J. 93(2):386-400, 2007. 